\title{
VARIABILIDADE DA PRECIPITAÇÃO E DO NÚMERO DE DIAS COM CHUVAS DE DUAS CIDADES DISTINTAS DA PARAÍBA
}

\author{
J. M. T. DINIZ \\ Programa de Pós-Graduação em Meteorologia - Universidade Federal de Campina Grande \\ julio_mannuel@hotmail.com
}

Artigo submetido em fevereiro/2013 e aceito em junho/2013

\section{RESUMO}

$\mathrm{Na}$ região Nordeste do Brasil é característica a significativa variabilidade espacial e temporal da precipitação pluvial, proveniente da atuação de diferentes sistemas meteorológicos. Em determinadas localidades do estado da Paraíba são comuns a ausência de chuvas durante períodos prolongados, ocasionando graves problemas para a sociedade e para os ecossistemas naturais. Neste sentido, o presente trabalho tem como objetivo a avaliação da precipitação e do número de dias com chuvas observados em duas cidades distintas da Paraíba. Os dados utilizados foram fornecidos pelo Instituto Nacional de Meteorologia e são provenientes de observações realizadas entre os anos de 2002 e 2011. A partir da análise dos resultados, verificou-se que o total de chuvas anuais e mensais observado em João Pessoa é bastante superior ao de Campina Grande. Em ambas as localidades, as chuvas apresentaram-se mais abundantes e regulares no primeiro semestre do ano, com valores máximos em torno do mês de Junho.

PALAVRAS-CHAVE: Nordeste do Brasil, precipitação pluvial, sistemas meteorológicos, número de dias com chuva.

\section{VARIABILITY OF RAINFALL AND NUMBER OF DAYS WITH RAIN IN TWO DISTINCT CITIES OF PARAÍBA}

\begin{abstract}
In the Northeast region of Brazil is characteristic the significant spatial and temporal variability of rainfall, due to the action of different meteorological systems. At certain locations in the state of Paraíba is common the absence of rain for prolonged periods, causing serious problems for society and natural ecosystems. In this sense, the present study aims to the evaluation of rainfall and number of days with rain observed in two distinct cities of Paraíba. The data has been provided by
\end{abstract}

the National Institute of Meteorology and came from observations performed between the years 2002 and 2011. From the analysis of the results it has been found that the annual and monthly rainfall total observed in João Pessoa is largely higher than that of Campina Grande. In both places, the rains were more abundant and regular in the first half of the year, with peaks around the month of June.

KEYWORDS: Northeast of Brazil, rainfall, meteorological systems, number of days with rain. 


\section{VARIABILIDADE DA PRECIPITAÇÃO E DO NÚMERO DE DIAS COM CHUVAS DE DUAS CIDADES DISTINTAS DA PARAÍBA}

\section{INTRODUÇÃO}

A precipitação pluviométrica é uma das variáveis meteorológicas de maior importância para a sociedade em consequência de sua influência direta sobre vários aspectos e atividades relacionadas ao homem (agricultura, pecuária, etc.). O excesso (enchentes) ou escassez (secas) de chuvas acarretam danos socioeconômicos e ambientais de grande magnitude. Uma importante ferramenta que auxilia na previsão desses eventos extremos é o monitoramento diário do regime pluviométrico sobre uma dada localidade (Vianello, 1991).

Segundo Arai et al. (2009), a precipitação pluvial possui significativa importância na caracterização do clima de uma determinada região, intervindo diretamente nas alternâncias de rendimento das culturas. Longos períodos de estiagem, além de ocasionarem danos à agricultura da região (principalmente em áreas que não são irrigadas), afetam também o nível de águas dos mananciais e reservatórios das usinas hidrelétricas, podendo gerar danos ao abastecimento urbano e à geração de energia elétrica (Silva et al., 2011).

Em relação a outros parâmetros meteorológicos, a distribuição espacial da precipitação sobre o globo é bastante complexa (Varejão-Silva, 2001). Observações indicam que, no geral, a precipitação alcança um máximo, tanto no continente quanto no oceano, próximo ao equador geográfico, consequência direta da ZCIT (zona de convergência intertropical). Esse fenômeno caracteriza-se pela convergência e ascensão do ar quente e úmido para níveis mais elevados da atmosfera, principais elementos que propiciam a origem das chuvas (Ahrens, 2001).

$\mathrm{Na}$ maioria das localidades situadas entre os trópicos, a precipitação ocorre com maior frequência durante os meses de verão. A estação chuvosa determina o período das atividades agrícolas, de modo que os fatores limitantes para uma produção agrícola satisfatória estão associados ao total e à intensidade das chuvas (Silva \& Rao, 2002). Nas regiões tropicais, qualquer que seja a época do ano, a precipitação pluvial é efetivamente utilizada para o desenvolvimento das plantas. Nas médias latitudes, porém, esse uso somente ocorre com as precipitações oriundas das estações isentas de congelamento (Ayoade, 1996).

A variação temporal da precipitação é nítida quando se observa que os valores obtidos durante longos períodos não indicam nenhum tipo de regularidade, principalmente nos trópicos, onde esse comportamento é mais acentuado. Um dos fatores responsáveis pela variabilidade da precipitação pluvial verificada na região Nordeste do Brasil está associado ao deslocamento meridional da ZCIT e ao tempo de sua permanência em latitudes mais ao sul do Equador. Além disso, sofre a influência direta de fenômenos atmosféricos e oceânicos de grande escala que se processam (simultaneamente ou não) sobre os Oceanos Pacífico e Atlântico Tropicais (Lucena et al., 2011).

No Nordeste do Brasil, região em que é comum a maior parte da população sobreviver a 
partir da agricultura de sequeiro (sem irrigação), o sucesso das culturas depende fortemente da quantidade e regularidade das chuvas (Silva et al., 2011). Conforme Chiaranda et al. (2012), o estudo a respeito dessa variável meteorológica é indispensável para o planejamento das atividades agrícolas, pois permite previsões mais consistentes e decisões mais confiáveis.

Visto que o entendimento do regime pluviométrico de uma determinada região pode trazer benefícios significativos para o setor agrícola, principalmente em situações nas quais a disponibilidade hídrica não é abundante na maior parte do tempo, o presente trabalho tem como objetivo analisar a variabilidade da precipitação e do número de dias com chuvas observados ao longo de uma década em duas regiões distintas da Paraíba.

\section{MATERIAIS E MÉTODOS}

Para a elaboração da presente pesquisa foram utilizados dados obtidos através de pluviômetros alojados em duas regiões distintas do estado da Paraíba, mais especificamente em João Pessoa $\left(7,11^{\circ} \mathrm{S} ; 34,86^{\circ} \mathrm{O} ; 7,4 \mathrm{~m}\right)$ e Campina Grande $\left(7,22^{\circ} \mathrm{S} ; 35,88^{\circ} \mathrm{O} ; 547,6 \mathrm{~m}\right)$. Essas cidades foram selecionadas em virtude de suas características climáticas específicas e também devido à consistência e disponibilidade das informações.

Os dados de precipitação e número de dias com chuvas utilizados neste trabalho são referentes aos anos de 2002 a 2011. Esses, por sua vez, foram submetidos a análise estatística, tendo em vista obter resultados científicos mais aprimorados. O desvio padrão e o coeficiente de variabilidade foram as ferramentas utilizadas a fim de verificar as variações das variáveis de estudo, cujos detalhes podem ser encontrados em Thurman (2012).

A metodologia utilizada na presente pesquisa é baseada na utilizada por Pereira et al. (2011) ao realizarem estudo semelhante acerca da variabilidade do número de dias com chuvas observados em pontos distintos do estado de Sergipe.

Segundo Kokoska (2012), o grau e o tipo de relação existente entre duas variáveis quaisquer podem ser quantificados através do chamado coeficiente de correlação ( $r$ ). Um dos objetivos deste estudo concentra-se em verificar, em ambas as localidades, a correlação entre o total de precipitação e o número de dias com chuvas. Para alcançar esse objetivo fez-se uso da expressão (Larson \& Farber, 2004):

$$
\mathbf{r}=\frac{\mathbf{N} \sum \mathbf{X Y}-\left[\left(\sum \mathbf{X}\right)\left(\sum \mathbf{Y}\right)\right]}{\sqrt{\left[\mathbf{N} \sum \mathbf{X}^{\mathbf{2}}-\left(\sum \mathbf{X}\right)^{2}\right]\left[\mathbf{N} \sum \mathbf{Y}^{2}-\left(\sum \mathbf{Y}\right)^{2}\right]}}
$$

De tal modo que $X$ e $Y$ são os valores assumidos pelas variáveis (total de precipitação e número de dias com chuvas, respectivamente) e $\mathrm{N}$ é o tamanho da série temporal.

Após verificar-se a correlação existente entre as variáveis de estudo, o próximo passo consiste em determinar a equação da reta que melhor se ajusta aos dados. Denomina-se essa reta de regressão e sua equação pode ser usada para prever o valor de uma das variáveis no desconhecimento da outra. A equação de uma reta de regressão para uma variável independente $x$ e uma variável dependente y é expressa da seguinte maneira: 


$$
\mathbf{y}=\mathbf{m x}+\mathbf{b}
$$

$\mathrm{Na}$ qual y é o valor previsto para a variável dependente a partir de determinado valor assumido por $\mathrm{x}$. Neste estudo, considera-se como variável independente o número de dias com chuvas e o total de precipitação como sendo a variável dependente. De acordo com Spiegel (1993), a inclinação da reta (m) e o valor da constante (b) são determinados da seguinte maneira:

$$
\begin{aligned}
& \mathbf{m}=\frac{\mathbf{N} \sum \mathbf{X Y}-\left[\left(\sum \mathbf{X}\right)\left(\sum \mathbf{Y}\right)\right]}{\mathbf{N} \sum \mathbf{X}^{\mathbf{2}}-\left[\left(\sum \mathbf{X}\right)^{\mathbf{2}}\right]} \\
& \mathbf{b}=\frac{\sum \mathbf{Y}}{\mathbf{N}}-\mathbf{m} \frac{\sum \mathbf{X}}{\mathbf{N}}
\end{aligned}
$$

Nas equações acima, as incógnitas $\mathrm{N}, \mathrm{X}$ e $\mathrm{Y}$ representam novamente o tamanho da série temporal e os valores assumidos pelas variáveis de estudo.

\section{RESULTADOS E DISCUSSÕES}

A partir das informações fornecidas pelo INMET, tornou-se possível analisar neste estudo o número de dias com chuvas e a precipitação de duas regiões distintas da Paraíba. A Figura-1 apresenta o total de precipitação anual observado ao longo de uma década nas cidades de João Pessoa e Campina Grande.

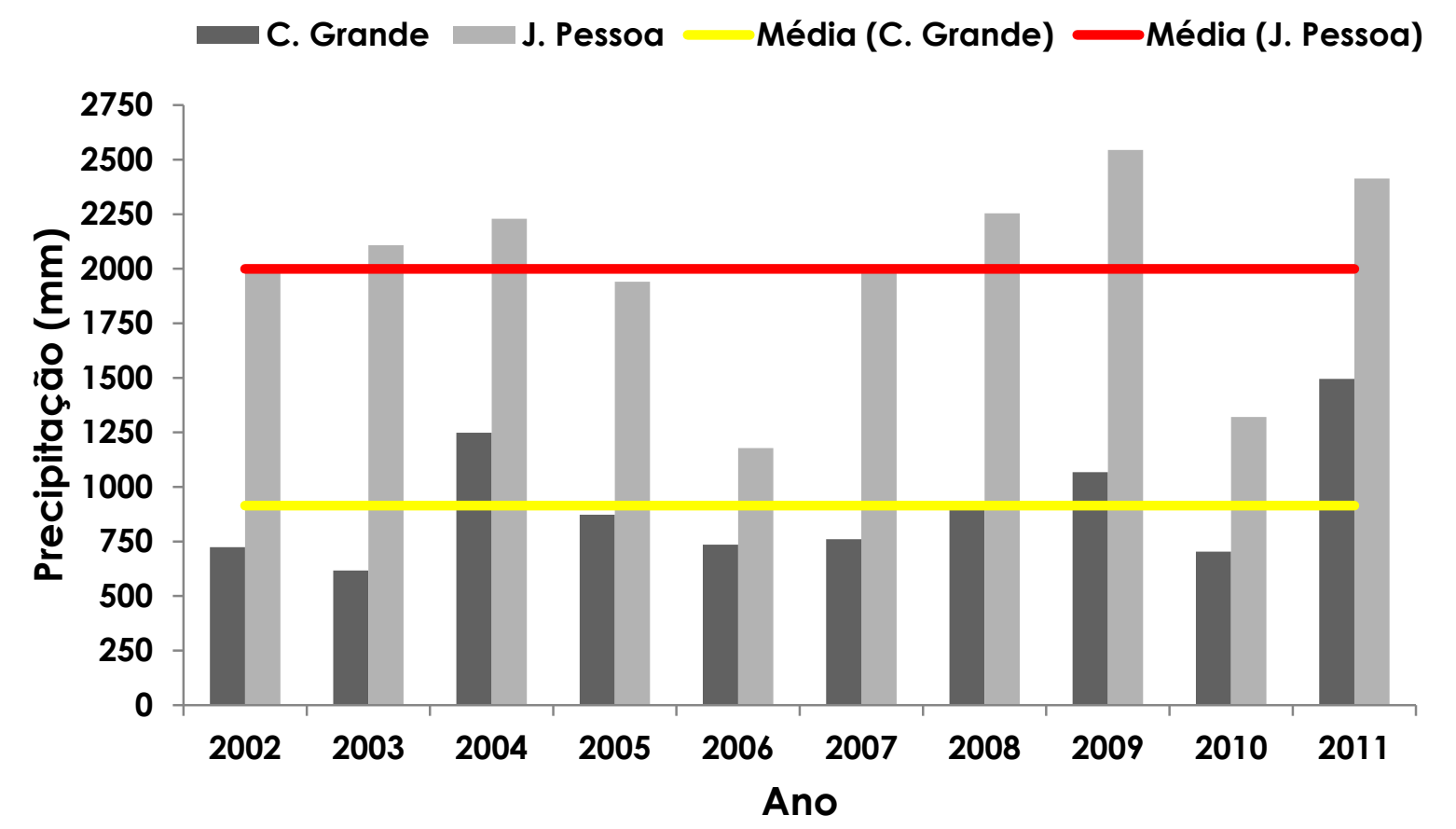

Figura 1 - Precipitação total e média anual de precipitação pluviométrica para as cidades de João Pessoa e Campina Grande.

Verifica-se claramente na Figura-1 que os totais anuais de precipitação em João Pessoa são significativamente mais elevados que os de Campina Grande. Esse comportamento já era esperado devido à localização geográfica de ambas as cidades, visto que João Pessoa situa-se no litoral e Campina Grande no agreste do estado em questão. Mediante os totais anuais, foi 
estimada a precipitação pluvial média anual e constatou-se que, para esse período, a obtida para João Pessoa é em torno de $46 \%$ superior à de Campina Grande.

Ao comparar a precipitação observada em João Pessoa nos anos de 2006 e 2010 com a respectiva média anual (linha vermelha), observa-se que o total de chuva nesses dois anos foi muito abaixo do valor esperado. Em contrapartida, precipitações acima da média anual foram verificadas em 2009 e 2011. Os demais anos analisados não podem ser classificados como secos ou chuvosos em virtude dos totais anuais se encontrarem em torno da média anual de precipitação.

Na cidade de Campina Grande, chuvas excessivas durante os anos de análise foram verificadas em 2004 e 2011, enquanto que abaixo da média foram as precipitações oriundas dos anos de 2003 e 2010. Devido ao fato de verificar-se, em ambas as localidades, excesso de chuva no ano de 2011 e escassez em 2010, acredita-se que esse comportamento é proveniente da influência direta de algum fenômeno meteorológico que atuou simultaneamente nas cidades de estudo, ocasionando, consequentemente, a intensificação e a redução da precipitação.

Informações detalhadas a respeito da precipitação mensal e anual de ambas as cidades podem ser observadas na Tabela-1 e na Tabela- 2 .

Tabela 1 - Médias (mensais e anuais), desvio-padrão, coeficiente de variação (CV) e valores extremos da precipitação pluviométrica, com os respectivos anos de ocorrência, na cidade de Campina Grande-PB, para o período de 2002 a 2011.

\begin{tabular}{cccccccc}
\hline Mês & $\begin{array}{c}\text { Média } \\
(\mathbf{m m})\end{array}$ & $\begin{array}{c}\text { D. Padrão } \\
(\mathbf{m m})\end{array}$ & $\begin{array}{c}\text { CV } \\
(\%)\end{array}$ & $\begin{array}{c}\text { Mínimo } \\
(\mathbf{m m})\end{array}$ & $\begin{array}{c}\text { Ano } \\
\text { Ocorrido }\end{array}$ & $\begin{array}{c}\text { Máximo } \\
(\mathbf{m m})\end{array}$ & $\begin{array}{c}\text { Ano } \\
\text { Ocorrido }\end{array}$ \\
\hline Janeiro & 69,4 & 73,5 & 106,0 & 1,0 & 2006 & 279,0 & 2004 \\
Fevereiro & 89,7 & 86,0 & 95,9 & 11,0 & 2008 & 244,6 & 2009 \\
Março & 106,4 & 57,5 & 54,1 & 21,2 & 2010 & 238,8 & 2008 \\
Abril & 96,6 & 53,1 & 55,0 & 23,9 & 2005 & 180,0 & 2011 \\
Maio & 134,3 & 89,7 & 66,8 & 27,1 & 2010 & 361,1 & 2011 \\
Junho & 155,2 & 51,2 & 33,0 & 97,8 & 2008 & 263,3 & 2005 \\
Julho & 112,4 & 84,6 & 75,3 & 42,1 & 2010 & 333,9 & 2011 \\
Agosto & 82,9 & 31,4 & 37,9 & 42,1 & 2004 & 138,6 & 2009 \\
Setembro & 29,5 & 23,3 & 78,9 & 2,3 & 2002 & 82,3 & 2007 \\
Outubro & 8,9 & 6,2 & 69,2 & 0,4 & 2009 & 22,6 & 2002 \\
Novembro & 14,3 & 14,8 & 103,8 & 0,8 & 2005 & 41,6 & 2002 \\
Dezembro & 14,7 & 13,2 & 90,3 & 2,7 & 2002 & 39,4 & 2005 \\
\hline Anual & 914,2 & 264,5 & 28,9 & 616,7 & 2003 & 1495,4 & 2011 \\
\hline
\end{tabular}

Tabela 2 - Médias (mensais e anuais), desvio-padrão, coeficiente de variação (CV) e valores extremos da precipitação pluviométrica, com os respectivos anos de ocorrência, na cidade de João Pessoa-PB, para o período de 2002 a 2011.

\begin{tabular}{cccccccc}
\hline Mês & $\begin{array}{c}\text { Média } \\
(\mathbf{m m})\end{array}$ & $\begin{array}{c}\text { D. Padrão } \\
(\mathbf{m m})\end{array}$ & $\begin{array}{c}\text { CV } \\
(\%)\end{array}$ & $\begin{array}{c}\text { Mínimo } \\
(\mathbf{m m})\end{array}$ & $\begin{array}{c}\text { Ano } \\
\text { Ocorrido }\end{array}$ & $\begin{array}{c}\text { Máximo } \\
(\mathbf{m m})\end{array}$ & $\begin{array}{c}\text { Ano } \\
\text { Ocorrido }\end{array}$ \\
\hline Janeiro & 108,5 & 91,5 & 84,3 & 7,2 & 2006 & 303,2 & 2004 \\
Fevereiro & 132,0 & 88,3 & 66,9 & 8,8 & 2008 & 260,1 & 2009
\end{tabular}




\begin{tabular}{cccccccc} 
Março & 160,0 & 87,1 & 54,5 & 30,9 & 2010 & 299,3 & 2008 \\
Abril & 255,2 & 129,3 & 50,7 & 125,1 & 2005 & 547,4 & 2009 \\
Maio & 321,2 & 142,3 & 44,3 & 111,7 & 2010 & 549,5 & 2005 \\
Junho & 420,6 & 137,3 & 32,7 & 248,7 & 2011 & 626,2 & 2003 \\
Julho & 263,7 & 129,4 & 49,1 & 96,3 & 2003 & 475,8 & 2004 \\
Agosto & 194,3 & 62,6 & 32,2 & 119,0 & 2006 & 326,4 & 2008 \\
Setembro & 64,1 & 25,9 & 40,4 & 13,4 & 2002 & 97,2 & 2003 \\
Outubro & 26,3 & 12,1 & 46,0 & 9,6 & 2011 & 44,9 & 2008 \\
Novembro & 24,8 & 16,3 & 65,9 & 4,1 & 2005 & 45,8 & 2009 \\
Dezembro & 29,3 & 20,5 & 70,0 & 5,1 & 2004 & 65,5 & 2003 \\
\hline Anual & 1999,8 & 416,8 & 20,8 & 1178,3 & 2006 & 2544,5 & 2009 \\
\hline
\end{tabular}

Observa-se, nas tabelas acima, que durante todo o ciclo anual as maiores médias mensais de precipitação são verificadas entre os meses de Maio e Julho, com o máximo ocorrendo no mês de Junho. A partir das médias mensais examina-se que, no geral, em ambas as localidades as chuvas são mais abundantes no primeiro semestre do ano. Após esse período inicia-se uma progressiva diminuição das chuvas, alcançando valores mínimos nos três últimos meses do ano. Como já se esperava em virtude dos totais anuais e pelo discutido anteriormente, os valores estimados para a precipitação média mensal de João Pessoa são superiores aos de Campina Grande.

Verifica-se também que os menores valores do coeficiente de variação da precipitação mensal estão associados a meses considerados chuvosos. Esse resultado indica certa homogeneidade nos totais de chuvas oriundos dos meses em torno de Junho.

Os resultados anteriores estão de acordo com estudos que indicam que o período de chuvas mais abundantes no leste do Nordeste do Brasil abrange os meses de Abril a Julho (Nobre \& Molion, 1988). A chuva desse período está associada à maior atividade de circulação de brisa que advecta bandas de nebulosidade para o continente, bem como também está relacionada aos distúrbios ondulatórios de leste, que são responsáveis pelas chuvas entre os meses de Maio e Agosto (Yamazaki \& Rao, 1977).

Considerando os resultados vistos anteriormente, recomenda-se à população que, caso necessário, a agricultura de sequeiro deve ser praticada no primeiro semestre do ano, em virtude da maior abundância e regularidade das chuvas nesse período.

Mediante o conhecimento do número de dias de chuva, foram estimadas as respectivas médias mensais e anuais. A Tabela-3 apresenta, para ambas às localidades, os valores médios e os coeficientes de variação do número de dias com chuva.

Tabela 3 - Médias (mensais e anuais) e coeficientes de variação (CV) do número de dias com chuva para as cidades de Campina Grande e João Pessoa.

\begin{tabular}{|c|c|c|c|c|}
\hline \multirow[t]{2}{*}{ Mês } & \multicolumn{2}{|c|}{ Campina Grande } & \multicolumn{2}{|c|}{ João Pessoa } \\
\hline & Média & CV (\%) & Média & CV (\%) \\
\hline Janeiro & 9,9 & 58,80 & 12,8 & 47,2 \\
\hline
\end{tabular}




\begin{tabular}{ccccc} 
Fevereiro & 9,9 & 38,45 & 12,3 & 33,7 \\
Março & 11,5 & 39,13 & 15,3 & 23,2 \\
Abril & 15,1 & 19,30 & 21,2 & 12,6 \\
Maio & 17,5 & 31,84 & 22,0 & 17,1 \\
Junho & 21,4 & 18,48 & 24,5 & 15,2 \\
Julho & 19,0 & 21,95 & 22,6 & 16,0 \\
Agosto & 18,1 & 23,24 & 21,7 & 15,0 \\
Setembro & 9,4 & 30,91 & 14,5 & 22,3 \\
Outubro & 5,5 & 31,75 & 10,2 & 33,6 \\
Novembro & 5,2 & 46,95 & 9,3 & 31,9 \\
Dezembro & 5,2 & 35,25 & 9,9 & 22,8 \\
\hline Anual & 147,7 & 7,53 & 196,3 & 7,07 \\
\hline
\end{tabular}

Na Tabela-3 é nítido que, em média, João Pessoa apresenta mais dias de chuva que Campina Grande. Nas duas localidades, os meses com menos dias de chuva são oriundos dos primeiros e últimos meses do ano, enquanto que o oposto é verificado em torno do mês de Junho. Em relação ao ciclo anual, João Pessoa possui em média cerca de 50 dias a mais de chuva que Campina Grande. Todos esses comportamentos refletem o total de precipitação mensal e anual, indicando algum tipo de correlação que será abordada, mais adiante, entre o número de dias com chuva e a precipitação total.

Ao analisar a tabela anterior, verifica-se que, em geral, as maiores variabilidades ocorrem nos períodos em que a precipitação média é baixa e as menores quando a mesma é elevada. Portanto, meses considerados secos, em virtude da baixa precipitação pluvial observada ao longo dos anos analisados, possuem, em relação à média, maior variação do número de dias com chuvas do que os períodos ditos chuvosos, ou seja, meses em que é característica a elevada precipitação apresentam certa regularidade quanto ao número de dias com chuvas. Conclui-se, consequentemente, que o coeficiente de variabilidade é inversamente proporcional ao total de chuvas (os maiores CV são observados no início e no fim de ano e os menores por volta do mês de Junho). Em estudo similar, Pereira et al. (2012) observaram um comportamento semelhante ao examinar a variabilidade do número de dias chuvas em todo o estado de Alagoas.

Uma das maneiras mais precisas de medir o tipo e o grau de uma correlação entre duas variáveis é por meio do cálculo do coeficiente de correlação (r). A Figura-2 apresenta a correlação e a reta de regressão para o total de precipitação e o número de dias com chuvas. 


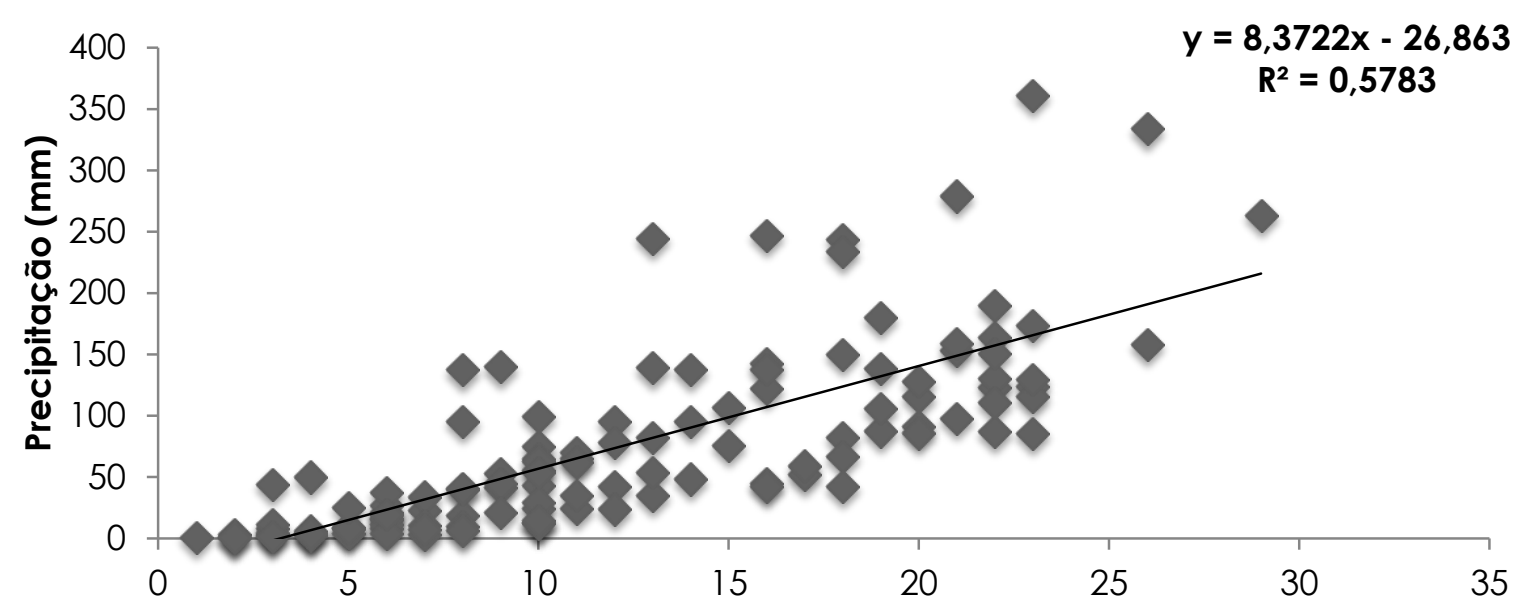

Número de dias com chuva

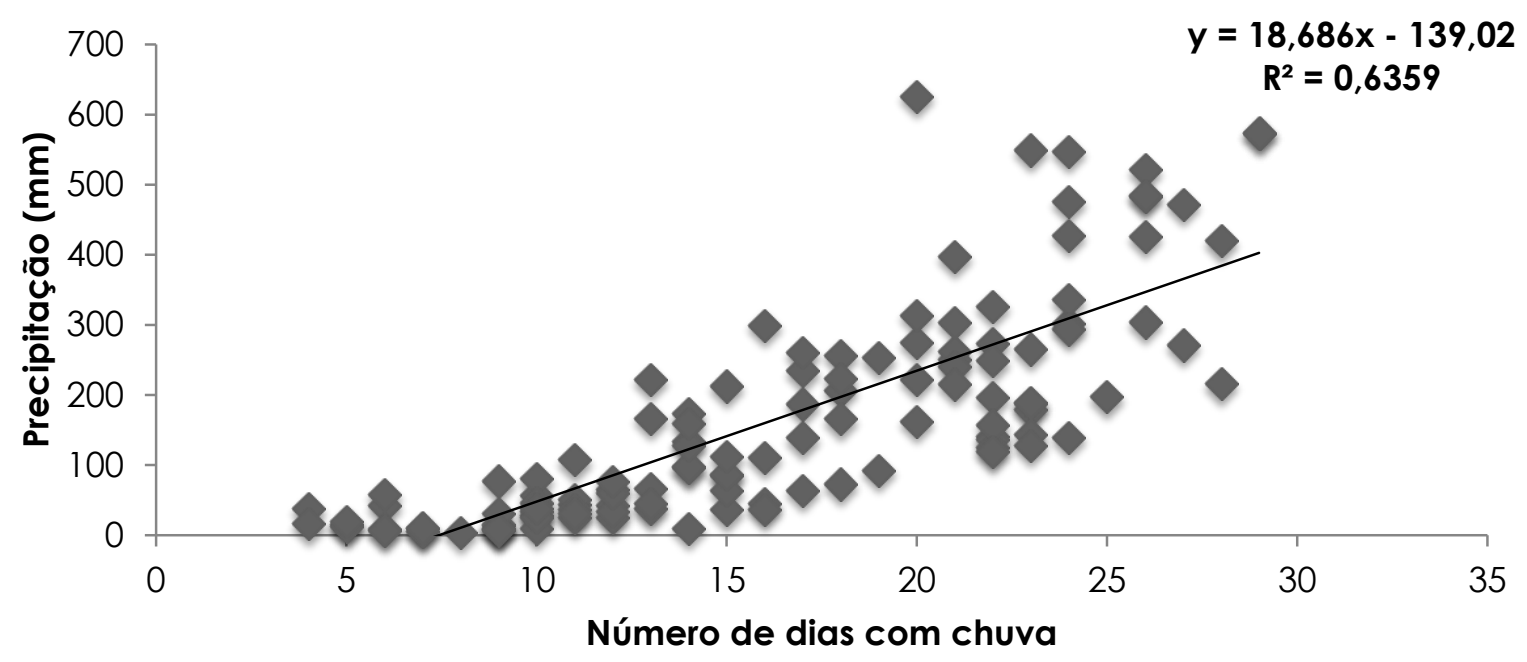

Figura 2 - Correlações entre o número de dias com chuva e o total de precipitação para Campina Grande (superior) e João Pessoa (inferior), obtidos fazendo uso de dados observados em ambas as localidades nos anos de 2002 a 2011.

Observa-se, a partir da Figura-2, que em primeira análise há uma correlação linear positiva entre o total de precipitação e o número de dias com chuvas, de modo que o coeficiente de correlação apresenta maior magnitude na cidade de João Pessoa $(r=0,7974)$ que em Campina Grande $(r=0,7604)$. Os baixos valores do coeficiente de determinação $\left(r^{2}\right)$, parâmetro utilizado para saber o grau de ajustamento do modelo estatístico aos dados coletados, indica que a equação da reta de regressão não representa significativamente a relação existente entre as variáveis de estudo. A fim de se obter um melhor ajuste, deve-se utilizar uma maior amostra de dados, com o intuito de verificar se o modelo mais eficaz a ser empregado é realmente o linear, além de serem aplicados critérios mais minuciosos para a coleta e tratamento das informações na busca de minimizar os erros amostrais.

\section{CONCLUSÃO}

A partir da análise dos resultados, tornou-se possível concluir que a precipitação anual e mensal em João Pessoa é significativamente superior à de Campina Grande. Em alguns anos, a 
precipitação anual sofre a influência de fenômenos meteorológicos e oceânicos adversos, que ocasionam a intensificação ou redução das chuvas.

Em ambas as localidades, a precipitação apresentou-se mais abundante no primeiro semestre do ano, com o valor máximo em torno do mês de Junho. Nos meses seguintes ocorreu uma progressiva diminuição das chuvas, de modo que os menores valores são observados em Setembro, Outubro, Novembro e Dezembro.

Verificou-se também que o coeficiente de variação do número de dias com chuvas é inversamente proporcional ao total de precipitação. Além disso, o número de dias com chuvas reflete os totais mensais e anuais de precipitação, de modo que ambas as variáveis apresentam satisfatória correlação.

\section{REFERÊNCIAS BIBLIOGRÁFICAS}

1. AHRENS, C. D. Essentials of Meteorology: An Invitation to the Atmosphere. 3a Ed. New York: Brooks Cole, 2001. 463 p.

2. ARAI, F. K.; PEREIRA, S. B.; GONÇALVES, G.; DANIEL, O.; PEIXOTO, P.; VITORINO, A. C. T. Espacialização da precipitação pluvial na Bacia do Rio Dourados. In: CONGRESSO BRASILEIRO DE CIÊNCIA DO SOLO, 2-7 ago., Fortaleza. Anais... Fortaleza: UFC, 2009. CD-ROM.

3. AYOADE, J. O. Introdução a climatologia para os trópicos. 4a Ed. Rio de Janeiro: Bertrand Brasil, 1996. 332 p.

4. CHIARANDA, R.; RIZZI, N. E.; COLPINI, C.; SOARES, T. S.; SILVA, V. S. M. Análise da precipitação e da vazão da bacia do Rio Cuiabá. Revista Brasileira de Ciências Agrárias, Recife, v. 7, n. 1, p. 117-122, 2012.

5. KOKOSKA, S. Introdução à estatística. Rio de Janeiro: LTC, 2012. 744 p.

6. LARSON, R.; FARBER, B. Estatística aplicada. 2a Ed. São Paulo: Prentice Hall, 2004. 476 p.

7. LUCENA, D. B.; GOMES FILHO, M. F.; SERVAIN, J. Avaliação do impacto de eventos climáticos extremos nos Oceanos Pacífico e Atlântico sobre a estação chuvosa no nordeste do Brasil. Revista Brasileira de Meteorologia, v. 26, n. 2, p. 297-312, 2011.

8. NOBRE, C. A.; MOLION, L. C. B. The climatology of drought prediction. Norwell: Kluwer Academic Publishers, 1988.

9. PEREIRA, E. R. R.; FREITAS, J. C.; ANDRADE, A. R. S.; SOUSA, V. G. Variabilidade do número de dias chuvosos no estado de Sergipe-SE. Revista Brasileira de Tecnologia Aplicada nas Ciências Agrárias, Guarapuava, v. 4, n. 1, p. 45-65, 2011.

10. PEReIRA, E. R. R.; FReITAS, J. C.; ANDRADE, A. R. S.; MACEDO, M. L. A.; SILVA NetO, M. F. Variabilidade do número de dias de chuva no estado de Alagoas. Revista Brasileira de Tecnologia Aplicada nas Ciências Agrárias, Guarapuava, v. 5, n. 1, p. 7-26, 2012. 
11. SILVA, S. A.; LIMA, J. S. S.; BOTTEGA, E. L. Variabilidade espacial da precipitação pluviométrica para o Estado do Espírito Santo utilizando métodos multivariados. Revista Brasileira de Ciências Agrárias, Recife, v. 6, n. 4, p. 703-709, 2011.

12. SILVA, V. P. R.; PEREIRA, E. R. R.; AZEVEDO, P. V.; SOUSA, F. A. S.; SOUSA, I. F. Análise da pluviometria e dias chuvosos na região Nordeste do Brasil. Revista Brasileira de Engenharia Agrícola e Ambiental, Campina Grande, v. 15, n. 2, p. 131-138, 2011.

13. SILVA, F. A. S.; RAO, T. V. R. Regimes pluviais, estação chuvosa e probabilidade de ocorrência de veranicos no Estado do Ceará. Revista Brasileira de Engenharia Agrícola e Ambiental, Campina Grande, v. 6, n. 3, p. 453-459, 2002.

14. SPIEGEL, M. R. Estatística. 3a Ed. São Paulo: Makron Books, 1993. 643 p.

15. THURMAN, P. W. Estatística. São Paulo: Saraiva, 2012. 232 p.

16. VAREJÃO-SILVA, M. A. Meteorologia e Climatologia. 2a Ed. Brasília: Gráfica e Editora Pax, 2001. 532p.

17. VIANELLO, R. L. Meteorologia básica e aplicações. 1ạ Ed. Viçosa: Imprensa Universitária, 1991. $449 \mathrm{p}$.

18. YAMAZAKI, Y.; RAO, V. B. Tropical cloudiness over the South Atlantic Ocean. Journal Meteorological Society of Japan, v. 55, p. 205-207, 1977. 